\title{
Learning from mistakes
}

\author{
LE Nicolle MD FRCP, Editor-in-Chief
}

$\mathrm{I}^{\mathrm{n}}$ $\mathrm{n}$ the present issue of the Journal, Patrick and his colleagues 1 (pages 330-336) describe an episode in the summer of 2003, immediately following the severe acute respiratory syndrome (SARS) epidemic, when an outbreak of respiratory illness occurred in a long-term care facility in Vancouver, and was initially reported by the National Microbiology Laboratory (NML) to be SARS. The local laboratories did not support the diagnosis and, subsequently, the NML diagnosis was acknowledged to be incorrect. The misdiagnosis, however, had an immediate negative impact by suggesting that SARS continued to be transmitted in Canada, raising the spectre of social and economic impacts recently experienced by Toronto. The episode also had a longer term negative impact on national and international perceptions of the reliability of the Canadian laboratory. Thus, a critical review of this episode to understand what happened and to avoid future errors is appropriate. Dr Patrick and his coauthors, including those from the NML, are to be congratulated for presenting this information to the Canadian infectious diseases and public health communities.

From the current perspective - over three years later - the events seem straightforward. An outbreak of a respiratory infection was identified in early July 2003 in a long-term care facility in Vancouver, just as the World Health Organization announced that human transmission of SARS coronavirus (SARS-CoV) had ceased. Over 50\% of residents and onethird of staff in the facility were affected. The staff members and most of the residents had a mild illness, with a few residents having more severe outcomes. This presentation was consistent with several well-recognized respiratory viruses known to cause outbreaks in long-term care facilities. As the report notes, the clinical presentation and spectrum of illness were not consistent with the clinical picture of SARS in older populations and health care workers, which had been well described over the previous six months.

The heightened surveillance for respiratory illness because of concerns for both SARS and avian influenza led to diligent collection of specimens for laboratory analysis. These included respiratory secretions, and blood, stool and autopsy specimens. Specimens were analyzed by the BC Centre for Disease Control, the British Columbia Cancer Agency's Genome Sciences Centre and the NML. The spectrum of diagnostic tests included culture, nucleic acid-based testing and serology. The laboratory diagnosis of SARS at that time was not well standardized, and there was relatively limited experience with the reliability and potential pitfalls of the different diagnostic tests used. At the BC laboratories, all specimens were PCR-negative for specific SARS sequences, but there were a few positives in a PCR test using a more broadly reactive $\mathrm{CoV}$ base region. Nucleic acid testing for human $\mathrm{CoV}$ ( HCoV)-OC43, a wellrecognized cause of respiratory outbreaks in long-term care facilities, was positive in all specimens tested. In the serology tests, the BC Centre for Disease Control found indeterminant results on a neutralizing antibody for SARS in some specimens, but subsequent confirmatory testing was negative for all. The national laboratory reported that one-quarter to one-third of specimens were PCR-positive for SARS-CoV in two different assays. They also later confirmed that some specimens were positive in a specific $\mathrm{HCoV}-\mathrm{OC} 43$ assay. The national laboratory reported a small number of specimens positive in an ELISA assay for SARS-CoV, but none were positive for neutralizing antibody on convalescent testing.

It is now recognized that some of the antigens used for antibody tests were not SARS-specific but reacted with other $\mathrm{CoVs}$, including $\mathrm{HCoV}-\mathrm{OC} 43$. Because the outbreak was caused by the OC43 CoV strain, positive results would be anticipated for these tests. More difficult to explain is the PCR-positive specimens for SARS-CoV at the national laboratory. This is assumed to be a result of contamination in the laboratory at a time when large numbers of specimens from SARS patients in the Toronto outbreak were being assayed.

The report by Patrick et al is instructive in summarizing the diagnostic testing strategies and presumed explanations for the inaccuracies. The expanding knowledge about SARS and other CoVs, together with standardization of diagnostic tests for these agents, has clarified some of the confusion in the initial identification of the etiological diagnosis for this episode. The NML has subsequently rigorously reviewed its testing procedures, and no further problems suggesting contamination have been identified.

This experience, of course, reinforces some well-known fundamentals of laboratory and clinical medicine. The first is that the more tests you do, the more likely you are to have false positives. At the time of these events, a variety of different tests were performed, and the operational parameters of many of these tests were not well described or fully understood. The other wellrecognized observation is that diagnostic test results do not exist in isolation - they are always interpreted in the context of relevant clinical and epidemiological observations. Illness in these longterm care facility residents was not consistent with SARS, where mortality rates in individuals over 50 years of age exceeds $50 \%$.

In retrospect, the rapid and intensive mobilization of three laboratories in Canada to address diagnostic testing for this outbreak and to clarify the confusion in test results is a positive reflection on our national capacity to respond to emerging pathogens and complex laboratory diagnostic problems. The challenge with sophisticated laboratory tests performed independently at multiple facilities using the same specimens is to ensure an effective dialogue among clinicians, public health practitioners and laboratorians to facilitate appropriate and timely interpretation of results. The Public Health Agency of Canada is a legacy of the SARS outbreak, and this BC CoV outbreak is one element that contributed to the formation of the agency. The Public Health Agency of Canada has established several forums to support effective communication among diagnosticians, public health personnel and clinicians. While the effectiveness of the new agency has not yet been tested by an event of the magnitude of SARS, some lessons may have been learned from past experience.

Department of Internal Medicine, University of Manitoba, Winnipeg, Manitoba

Correspondence: Dr LE Nicolle, Department of Internal Medicine, University of Manitoba, Health Sciences Centre, GG443 - 820 Sherbrook

Street, Winnipeg, Manitoba R3A 1R9. Telephone 204-787-7029, fax 204-787-4826, e-mail nicolle@cc.umanitoba.ca 


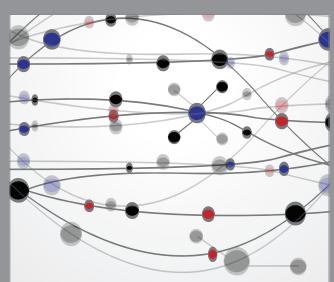

The Scientific World Journal
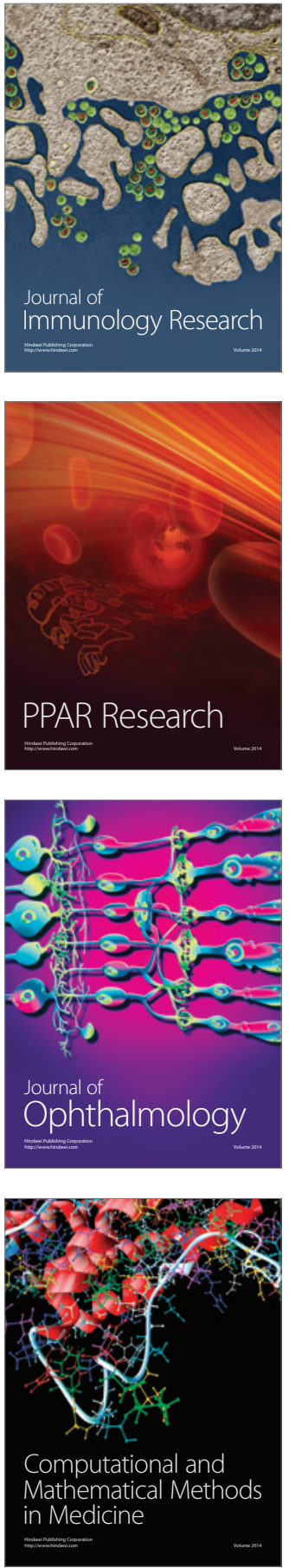

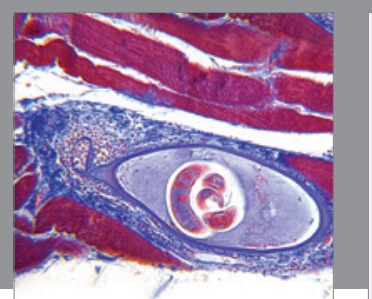

Gastroenterology Research and Practice

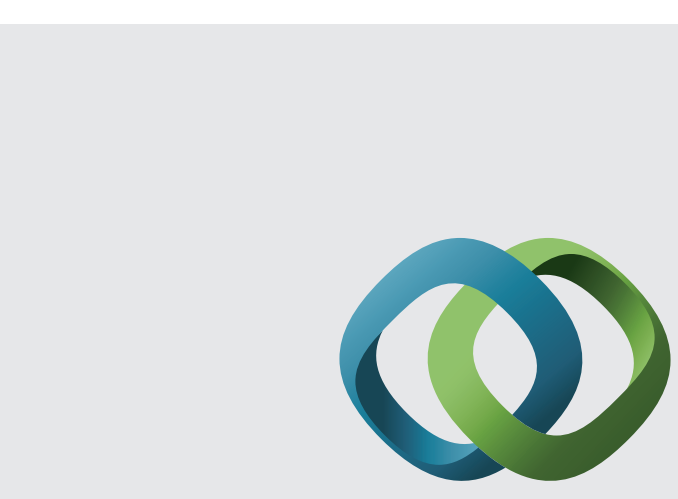

\section{Hindawi}

Submit your manuscripts at

http://www.hindawi.com
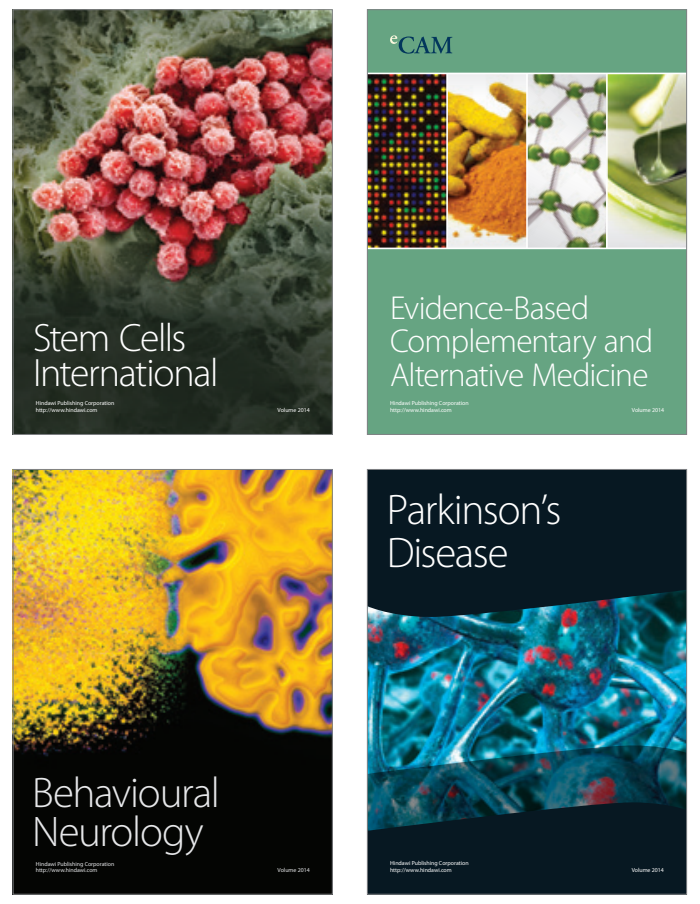
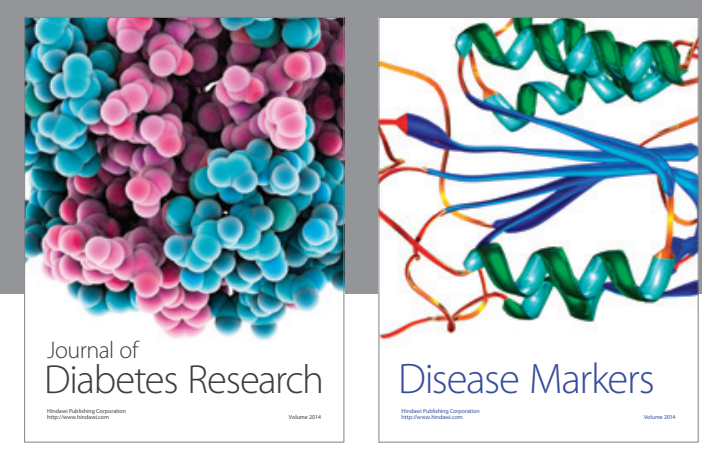

Disease Markers
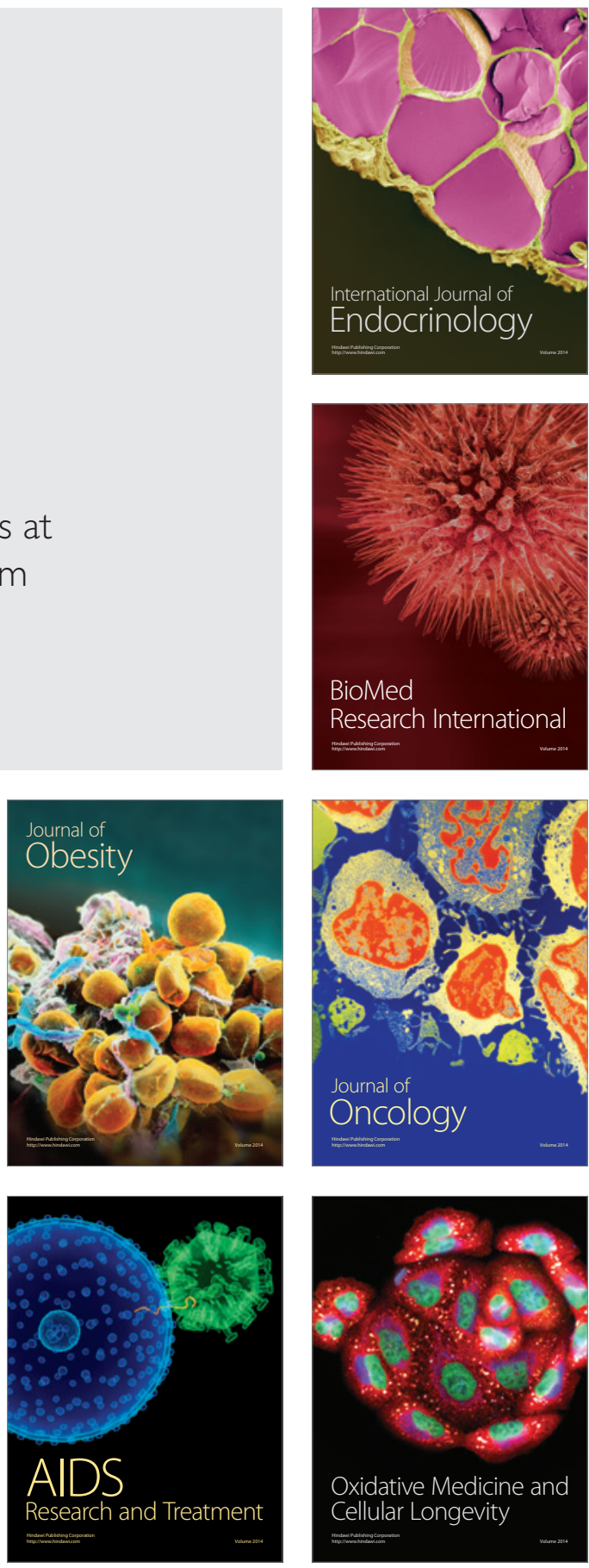